\title{
Sclerosing Mesenteritis: Clinical Presentation, Imaging Findings, and Treatment
}

Jennifer Nauheim, BSc and Rose Onyeali, MD

\section{CASE PRESENTATION}

A 67-year old male with a history of myocardial infarction status post percutaneous coronary intervention and stage IV bladder cancer status post radical cystoprostatectomy with ileal neobladder reconstruction and chemotherapy (cisplatin and gemcitabine) presented with progressive, severe epigastric and lower abdominal pain associated with nausea. The pain had intensified over the previous week and was associated with a recent fifteen pound weight loss in the setting of poor oral intake. He denied nausea or diarrhea.

His physical exam was notable for abdominal tympany and tenderness. His labs were notable for mild hyperkalemia (potassium $5.0 \mathrm{mmol} / \mathrm{L}$ ), normal white blood cell count, lipase of $28 \mathrm{U} / \mathrm{L}$, normal liver function tests, and positive 2+ leukocyte esterase and 1+ blood in his urine. Upper endoscopy showed a small Schatzki's ring in the distal third of the esophagus with small hiatal hernia and gastritis. On CT, there was thickened, indurated mesentery and a mesenteric mass (Figure 1) with abdominal lymphadenopathy and a new adrenal nodule.

\section{EPIDEMIOLOGY \& CLINICAL PRESENTATION}

Sclerosing mesenteritis, also known as mesenteric panniculitis, is part of a spectrum of idiopathic primary inflammatory and fibrotic processes that affect the mesentery and is characterized by inflammation of mesenteric fat. Although its etiology is unclear, it has been associated with previous abdominal surgery, autoimmunity, paraneoplastic syndromes, ischemic injury, and infection. Sclerosing mesenteritis is also associated with a high prevalence of coexisting malignancies and future cancer development. ${ }^{1}$ In a study evaluating 7620 patients presenting with a chief complaint of abdominal pain, the prevalence was $0.6 \%$.

The presentation of sclerosing mesenteritis is variable. In a series of 68 patients with sclerosing mesenteritis, $75 \%$ had abdominal pain, $26 \%$ had nausea/vomiting, $20 \%$ had anorexia and weight loss, and 20\% had altered bowel habits. ${ }^{3}$ In an 84-patient cohort, only $35 \%$ had abdominal pain, and $20 \%$ had an incidental abdominal mass on exam. ${ }^{2}$ The duration of symptoms ranged from 24 hours to 2 years. ${ }^{2}$

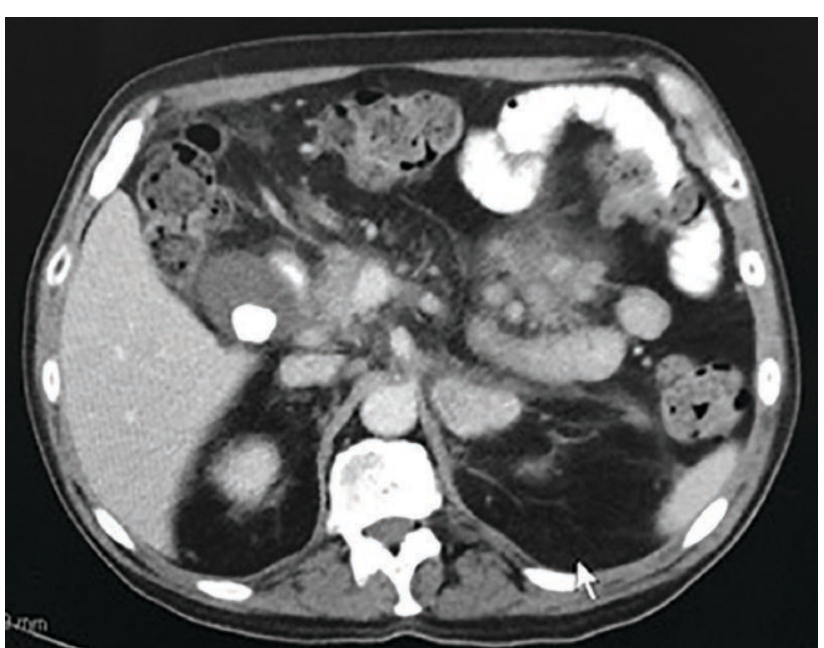

Figure 1. CT of the patient's abdomen showing a thickened, indurated mesentery

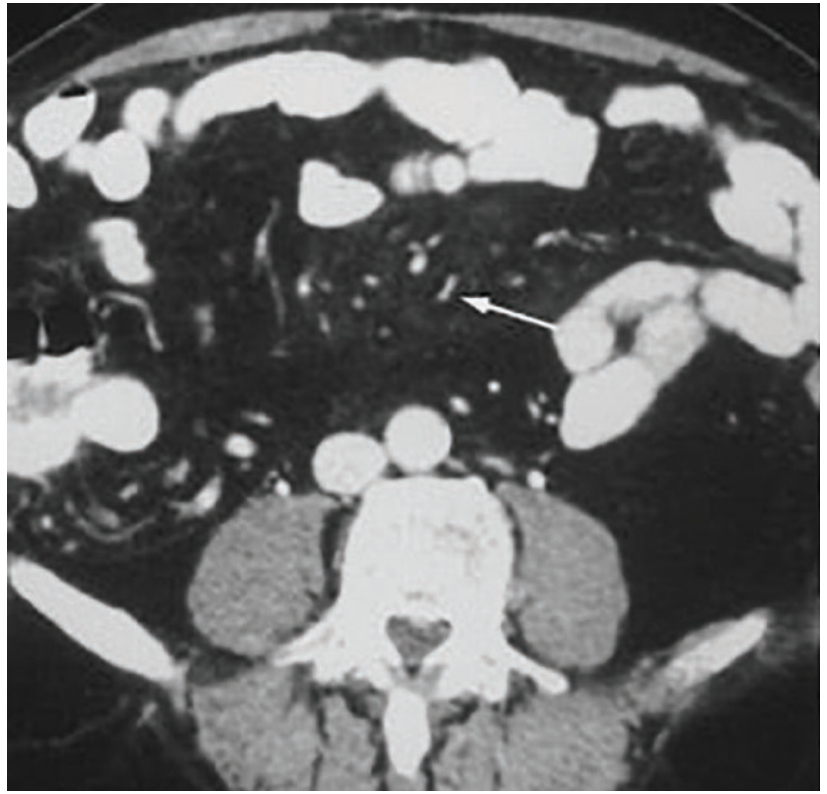

Figure 2. Fat ring sign (white arrow) seen on CT abdomen.

Up to $20 \%$ of patients with sclerosing mesenteritis develop complications, often intestinal obstruction from mass effect. ${ }^{3}$ Other complications include mesenteric vascular occlusion, chylous ascites, and adverse effects from medications used to treat sclerosing mesenteritis. 


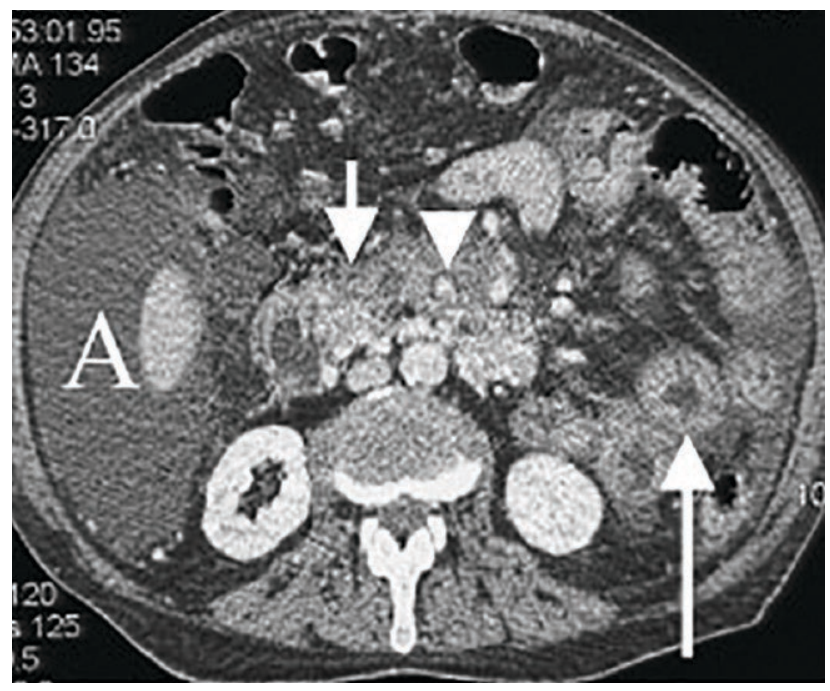

Figure 3. Tumor pseudocapsule (white arrow) seen on CT abdomen.

\section{DIAGNOSIS}

The diagnosis of sclerosing mesenteritis is made by histology and radiographic findings, with noninvasive imaging being preferred over surgical sampling. Dual phase computed tomography is the most sensitive imaging modality for detecting sclerosing mesenteritis.

Sclerosing mesenteritis typically presents on imaging as, in decreasing order of frequency, diffuse mesenteric thickening, a single mass with an average size of $10 \mathrm{~cm}$, or multiple masses. "Misty mesentery," haziness and stranding in the mesenteric fat, can be observed in sclerosing mesenteritis, but is not a specific imaging finding. The "fat ring sign," fat around mesenteric vessels spared from the changes of mesenteric panniculitis, is seen in 56-90\% of cases (Figure 2). A tumoral pseudocapsule is ocassionally seen (Figure 3). These latter two findings are not seen in other mesenteric diseases such as lipoma, lymphoma, or liposarcoma. ${ }^{4}$

\section{TREATMENT}

The preferred first-line therapy for sclerosing mesenteritis includes glucocorticoids in combination with tamoxifen, although glucocorticoids alone or with colchicine/ azathioprine may be beneficial. ${ }^{5}$ Patients with greater inflammatory components respond best to these regimens. A response to hormonal therapy with tamoxifen and progesterone has been reported as well. ${ }^{6}$ In refractory cases, thalidomide has been used.

\section{OUTCOME \& DISCUSSION}

The patient was started on tamoxifen and steroids for his sclerosing mesenteritis, but a biopsy of the enlarged abdominal lymph nodes and the adrenal nodule demonstrated worsening metastatic malignancy. Medical oncology advised that the patient discontinue tamoxifen and taper the steroids because of a lack of improvement in his abdominal pain. The patient received a celiac nerve block as an outpatient without any complications.

The differential diagnosis of a patient presenting with abdominal pain is broad. The differential for this patient after imaging included idiopathic sclerosing mesenteritis in the setting of metastatic bladder cancer, constipation secondary to opioid pain medications, narcotic bowel syndrome, pancreatitis, and worsening metastatic disease. It is likely that this patient's abdominal pain was multifactorial: his initial CT of his abdomen showed evidence of sclerosing mesenteritis with abdominal lymphadenopathy and an adrenal mass that were found on biopsy to be metastatic foci.

\section{REFERENCES}

1. Van Putte-Katier N, Van Bommel EFH, Elgersma OE, et al. Mesenteric panniculitis: prevalence, clinicoradiological presentation and 5-year follow-up. The British journal of radiology 2014;87(1044):20140451.

2. Daskalogiannaki M, Voloudaki A, Prassopoulos P, et al. CT evaluation of mesenteric panniculitis: prevalence and associated diseases. American Journal of Roentgenology 2000;174(2):427-431.

3. Sharma, P, Yadav S, Needham CM, Feuerstadt P. Sclerosing mesenteritis: a systematic review of 192 cases. Clinical journal of gastroenterology 2017;10(2):103-111.

4. Emory T, Monihan JM, Carr N, Sobin L. Sclerosing mesenteritis, mesenteric panniculitis and mesenteric lipodystrophy: a single entity? The American journal of surgical pathology 1997;21(4):392-398.

5. Akram S, Pardi DS, Schaffner JA, Smyrk TC. Sclerosing mesenteritis: clinical features, treatment, and outcome in ninety-two patients. Clinical Gastroenterology and Hepatology 2007:5(5):589-596.

6. Mazure R, Marty PF, Niveloni S, et al. Successful treatment of retractile mesenteritis with oral progesterone. Gastroenterology 1998:114(6):1313-1317. 